\title{
LAS-NCS: A Laser-Assisted Spintronic Neuromorphic Computing System
}

\author{
Hooman Farkhani, Member, IEEE, Ioan Lucian Prejbeanu, and Farshad Moradi, Senior Member, IEEE
}

\begin{abstract}
In spintronic-based neuromorphic computing systems (NCSs), the switching of magnetic moment in a magnetic tunnel junction (MTJ) is used to mimic biological neuron firing. However, the high currents required for MTJ switching will lead to a large energy consumption and low speed operation of the MTJ-based NCSs. In this paper, the energy consumption and the delay of the proposed MTJ-based NCS are reduced by thermally assisting the MTJ switching through a microwatt nanosecond laser pulse. Simulation results show $85.7 \%, 84 \%$ and $97.8 \%$ improvements in energy consumption, delay and energy-delay product, respectively, for the laser-assisted spintronic NCS (LAS-NCS) by heating the MTJs up to $127^{\circ} \mathrm{C}$. The energy consumption of the laser pulse for heating up the MTJ stack is 1.2X-2.4X lower than Joule heating method. Moreover, in contrast with Joule heating method, the LAS-NCS does not require MTJ with modified structure.
\end{abstract}

Index Terms - neuromorphic computing system, laser, energy efficient, COMSOL multiphysics, MTJ

\section{INTRODUCTION}

The grand challenge of exascale computing, $10^{18}$ operations/second, calls for a dramatic change in hardware of the current petascale supercomputers. A paradigm shift is required to tackle the issue of processing the huge amount of images and vidoes that is the most time and power consuming task for the existing Von-Neumann computing machines (VNC). The most promising solution is the brain-inspired systems, so called neuromorphic computing systems (NCS), which overcomes the limitation of the word-at-a-time thinking of the VNCs by massive parallel data processing similar to the brain [1-5]. An NCS includes many parallel processors (neurons) communicating using simple messages (spikes) through programmable memory units (synapses). Current implementation of NCSs using CMOS technology is area and power inefficient [6]. Such inefficiencies have driven a significant effort to investigate beyond-CMOS NCSs. The spin-based devices integrated with electronics (i.e. spintronics) have opened a door for designers to implement low-power high-density NCSs. In spintronic-based NCSs, a common way to mimic neuron firing is magnetic reversal of free layer magnetic moment in a magnetic tunnel junction (MTJ). However, it needs a high bias current $(>100 \mu \mathrm{A})$,

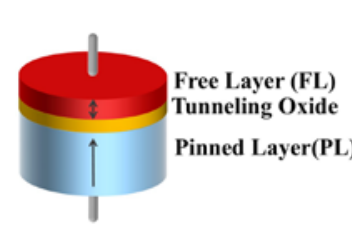

(a)

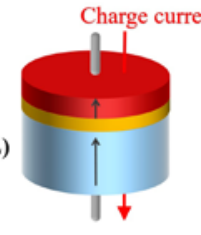

Low resistance

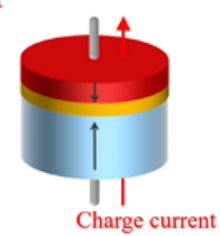

High resistance (b)

which leads to a huge gap between spintronic-based NCS and brain in terms of energy consumption and speed. Hence, there is a crucial need for decreasing the bias current in spintronic-based NCSs.

In this paper, benefiting from heating up the MTJ with a microwattnanosecond laser pulse, for the first time, we propose to design a laserassisted spintronic NCS (LAS-NCS) to improve energy-delay product of the state-of-the-art NCSs by at least $97.8 \%$; filling the huge gap of power and speed between the Brain and the NCSs.

\section{STATE OF THE ART}

The schematic of the MTJ is shown in Fig. 1 (a). It consists of a pinned layer (PL) with fixed magnetization and a free layer (FL) with changeable magnetization direction, which are separated by a tunneling oxide layer such as $\mathrm{MgO}$, AlOx etc. The resistance of the MTJ is determined by the relative magnetization direction of two ferromagnetic layers (Fig. 1 (b)). When the magnetization directions of FL and PL are parallel (P-state) or anti-parallel (AP-state), the MTJ resistance is low or high, respectively. In order to switch the magnetization direction of the FL, a spin-polarized current has to flow through the MTJ. This is done by applying an appropriate voltage to the terminals connected to the FL and PL and controlling the current direction. When a sufficient current flows from FL to PL, the MTJ switches to P-state. However, a sufficient reverse current (PL to FL) switches the MTJ state to the AP-state. In the MTJ-based NCS, the magnetization reversal of the FL is used to mimic biological neuron firing [6]. Thus, the MTJ state can be determined through evaluating its resistance using a read circuitry.

\section{A. Thermally Assisted Magnetization Switching}

Thermally assisting the MTJ is used to improve the FL switching in two different ways including creating a temperature gradient between FL and PL (Seebeck effect - SE) [7-9] and heating the MTJ above FL blocking temperature $\left(\mathrm{T}_{\mathrm{B}}\right)$ [10-12]. In SE method, using a temperature gradient across the MTJ, a pure spin current will be injected to FL, which switches the MTJ state [9]. Noted, in NCS application, heating up the MTJs should not lead to MTJ switching, but it just should assist switching. Hence, the SE method, in its original form, cannot be used in NCSs. In the second method, called thermally assisted switching (TAS), there is a need to use a modified type of MTJ. The FL consists of a ferromagnetic layer pinned with a low $\mathrm{T}_{\mathrm{B}}$ antiferromagnet (AF), such as FeMn $\left(90-160^{\circ} \mathrm{C}\right)$. The PL is a SyF pinned by an AF with high $\mathrm{T}_{\mathrm{B}}$, such as PtMn $\left(350^{\circ} \mathrm{C}\right)$ [11]. In standby mode, the FL presents a very high thermal stability, because it is pinned by the low $T_{B}$ antiferromagnet. Then, during switching phase, the stack heats up to $200^{\circ} \mathrm{C}$ in order to ease the FL switching [10]. In TAS, Joule heating is used to heat the MTJ junction above the blocking temperature of AF layer by passing an extra current through it. However, there are still some important challenges regarding TAS including inability to work with typical MTJs, power efficiency and low speed.

I. L. Prejbeanu is with University Grenoble Alpes CEA-CNRS, SPINTEC, Grenoble Cedex F-38000, France (e-mail: ioan-lucian.prejbeanu@cea.fr).

This work was supported by the Marie Sklodowska-Curie Individual Fellowship (IF) under contract number 751089.

H. Farkhani, and F. Moradi are with the Integrated Circuits and Electronics Laboratory, Department of Engineering, Aarhus University, 8000 Aarhus, Denmark (e-mail: farkhani@eng.au.dk; moradi@eng.au.dk). 
TABLE I. MTJ CHARACTERISTICS

\begin{tabular}{l|l}
\hline \hline Free Layer Dimensions & $\pi \times(30 \mathrm{~nm})^{2} \times(1.5 \mathrm{~nm})$ \\
\hline Oxide Thickness $\left(\mathrm{T}_{\mathrm{MgO}}\right)$ & $1.4 \mathrm{~nm}$ \\
\hline Saturation Magnetization $\left(\mathrm{M}_{\mathrm{S}}\right) @ 27^{\circ} \mathrm{C}$ & $1.1 \times 10^{8} \mathrm{~A} / \mathrm{m}$ \\
\hline FL Curry temperature & $1100 \mathrm{~K}$ \\
\hline Damping Factor $(\alpha)$ & 0.008 \\
\hline Gyromagnetic Factor $(\gamma)$ & $17.6 \mathrm{GHz} / \mathrm{Oe}$ \\
\hline
\end{tabular}

\section{B. MTJ Switching Behavior at Elevated Temperatures}

In order to model the dynamic behavior of the FL magnetic moment during MTJ switching, the Verilog-A model of [13] is used. In this model, the dynamic behavior of the FL magnetic moment is determined using Landau-Lifshitz-Gilbert-Slonczewski (LLGS) equation as follows [13]:

$$
\frac{\left(1+\alpha^{2}\right)}{|\gamma|} \frac{\partial \widehat{m}}{\partial t}=-\widehat{m} \times \vec{H}_{E F F}-\alpha \widehat{m} \times \widehat{m} \times \vec{H}_{E F F}
$$

where $\gamma, \alpha$ and $\widehat{m}$ are the gyromagnetic ratio, Gilbert damping factor and magnetization of FL, respectively. $\vec{H}_{E F F}$ is the effective magnetic field acting on FL described by $\vec{H}_{E F F}=\vec{H}_{U A}+\vec{H}_{e x}+\vec{H}_{S T T}+\vec{H}_{T F}$, where $\vec{H}_{U A}, \vec{H}_{e x}, \vec{H}_{S T T}$ and $\vec{H}_{T F}$ are uniaxial anistropy field, external magnetic field, current induced spin-transfer torque field and thermal fluctuations field, respectively. The effect of temperature on the dynamic behavior of the FL magnetic moment and thermal fluctuations effects are not included in this model. Hence, we added $\vec{H}_{T F}$ and temperature effect to this model in order to explore the thermally assisted MTJ-based NCS characteristics. Increasing the temperature affects the switching behavior of the MTJ through decreasing the magnetization saturation (MS) of the FL, decreasing the resistance of the MTJ and increasing the dispersion of the switching time due to higher thermal fluctuations.

Magnetization saturation: It is shown theoretically [14] and experimentally [15] that the dependency of MS can be well described by Bloch's law as $M_{S}(T)=M_{S}(0)\left(1-\left(T / T^{*}\right)^{\frac{3}{2}}\right) . T$ is the absolute temperature in Kelvin and $M_{S}(0)$ is the saturation magnetization at $0 \mathrm{~K}$, and $T^{*}$ is a fitting factor. Equation of $M_{S}$ shows that increasing the temperature decreases $\mathrm{M}_{\mathrm{S}}(\mathrm{T})$. This leads to a degradation of the uniaxial anisotropy field, which eases the FL magnetic switching.

Resistance: Two tunneling mechanisms contribute to the MTJ resistance including electron spin-polarized direct elastic tunneling and spin independent tunneling. The total conductance of the MTJ can be described as $G(\theta)=G_{T}\left[1+P_{1} P_{2} \cos \theta\right]+G_{S I} . \theta$ is the angle between the magnetization of the FL and the PL. $\mathrm{P}_{1}$ and $\mathrm{P}_{2}$ are the effective tunneling spin polarization of the magnetic layers. $\mathrm{G}_{\mathrm{T}}$ is the pre-factor for direct elastic tunneling. The $\mathrm{P}_{1}, \mathrm{P}_{2}$ and $\mathrm{G}_{\mathrm{T}}$ parameters are temperature-dependent. Elevating the temperature increases $\mathrm{G}_{\mathrm{T}}$ and reduces $P_{1}$ and $P_{2}$ [16]. As a result, $R_{P}$ is almost independent of temperature while $\mathrm{R}_{\mathrm{AP}}$ reduces approximately linearly with temperature. This has been experimentally shown in [16-18].

Thermal fluctuations: To add the effect of temperature on thermal fluctuations, a random fluctuating field $\vec{H}_{T F}$ is added to the $\vec{H}_{E F F}$. The $\mathrm{x}$, y and z component of the $\vec{H}_{T F}$ drawn from an uncorrelated Gaussian distribution with zero mean and $\sqrt{\left(2 \alpha k_{B} T\right) /\left(\gamma M_{S} V \Delta t\right)}$ standard deviation [19-21]. $\alpha, \mathrm{k}_{\mathrm{B}}, \gamma, \mathrm{V}$ and $\Delta \mathrm{t}$ are Gilbert damping parameter, Boltzmann's constant, gyromagnetic ratio, the volume of the FL and the integration time step. Elevating the temperature increases the dispersion of $\vec{H}_{T F}$, which leads to a higher switching probability. In order to benchmark the MTJ model, the experimental data of [17] is used (Table I). The MTJ structure in [17] is shaped circular with differrent diameters (D). Here, the experimental data of $\mathrm{D}=60 \mathrm{~nm}$ is used.

All in all, increasing the MTJ temperature decreases its $M_{S}$ and $R_{A P}$ which lead to lower energy consumption of the MTJ switching. Moreover, increasing the temperature increases the thermal fluctuation that increases the probability of switching (decreases the energy consumption of switching.

\section{Memristor Behavior at Elevated Temperature}

Memristor (memory resistor) is a passive two-port element with variable resistance. The resistance change of memristor depends on the total charge passing through it [28]. A unique feature of memristor is that it keeps its last resistance after cutting off the voltage/current source (it has a memory). The conduction mechanism of the memristor can be modeled by two parallel conduction mechanisms including hopping conduction and Schottky thermionic emission [22] as follows:

$$
I_{\text {total }}=\underbrace{2 e l v_{p h} N k_{B} T e^{\left(-\frac{W}{k_{B} T}\right)} e^{\left(-\frac{2 l}{\zeta}\right)} \sinh \left(\frac{q l F}{k_{B} T}\right)}_{I_{\text {hop }}}+\underbrace{A T^{2} e^{\left(-\frac{\phi_{B 0}-\beta \sqrt{F}}{k_{B} T}\right)}}_{I_{S c h}}
$$

where, $k_{B}$ is the Boltzmann constant, $l$ is the hopping distance, $W$ is the hopping energy, $\zeta$ is the wave function localization, $F$ is the applied field (converts from V), $T$ is the temperature, $v_{p h}$ is the vibrational phonon frequency, $A$ is the reduced effective Richardson constant multiplied by active device area, $\phi_{B 0}$ is the barrier height, and $\beta$ is the barrier lowering factor. $N$ is proportional to the density of electrons in the conduction path multiplied by the relevant conducting area. Based on this model, which is well-fitted with experimental results, the temperature dependence of memristor resistance can be divided into two regions called cold and hot regions [22]. In the cold region ( $\mathrm{T} \leq 350 \mathrm{~K}$ ), the state-dependent hopping conduction is dominant and the resistance of memristor is almost temperature insensitive. In the hot region, however, the Schottky emission of electrons determines the hot current and the memristor's resistance rapidly decreases with raising the temperature [22]. Noted, the trend of cold region (temperature independent) and hot region (temperature dependent) is independent of initial resistance state of memristor [22]. However, the amount of resistance change of memristor in hot region depends on memristor's initial resistance.

\section{Proposed Laser Assisted Neuromorphic Computing System}

Our novel envisioned LAS-NCS is shown in Fig. 2, which is a crossbar array of programmable memristors (196x10) as synapses and the MTJs as neurons, assisted thermally by a narrow laser-pulse. The resistance of the memristors can be tuned using an electric signal applying to them. The NCS operation can be divided into two phases including stimulation and recovery phases. In the stimulation phase, the crossbar array sums weighted input currents passing them to the MTJs, which are already set in AP-state. In case, the weighted input currents are sufficiently large, the MTJ state will be switched to P-state

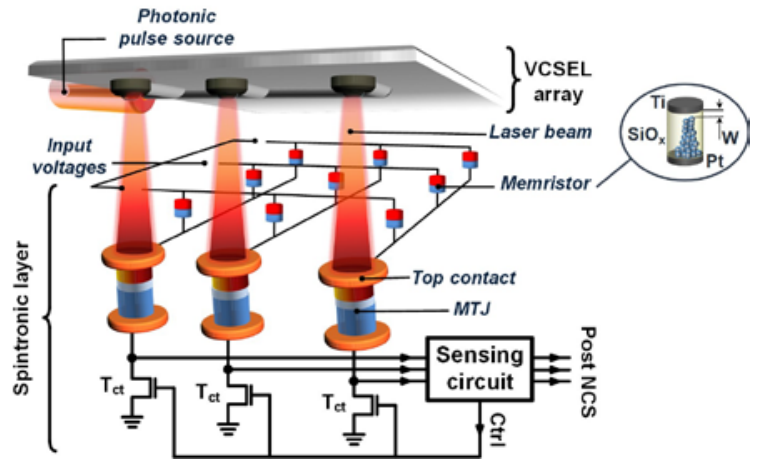

Fig. 2. The schematic view of the novel thermally assisted MTJ-based NCS. The MTJ and memristor act as neuron and synapse, respectively. 
that will be immediately detected by a sensing circuit [23], and translated to neuron's firing. Immediately after MTJ switching detection, the recovery phase begins. In the recovery phase, the input corresponding to the switched neuron will be activated in the post NCS. Then, the switched neuron will be switched back to the AP-state by passing a relatively high current. In our approach, the energy consumption and the delay of the MTJ switching will be lowered significantly by increasing the temperature of the MTJ using a nanosecond laser pulse. In fact, increasing the temperature of the MTJ will decrease the MTJ energy barrier, which leads to a low energy and fast MTJ switching. As a result, the energy consumption and the delay of the NCS decreases in both phases. The neuron consists of the MTJ and an activating transistor in series. Considering the fact that the transistor acts as a switch, heating it up has no significant impact on the overall performance of the LAS-NCS.

\section{A. Interaction between Laser and MTJ}

The cylindrical MTJ stack of [17] with $D=60 \mathrm{~nm}$ is used in the simulations. The shape, material and sizing of each layer is exactly similar to [17]. The laser beam is illuminated on the top electrode of the MTJ stack to heat up the overal temperature of the MTJ and its corresponding memristors. Hence, the top electrode should absorb the maximum laser energy in order to achieve maximum efficiency. The electrodes are made of $\mathrm{Au}$ with a thickness of $15 \mathrm{~nm}$. A nanosecond laser with $355 \mathrm{~nm}$ wavelength is used to decrease the transmissivity of electrode. The optical transmittance and reflectance of the electrodes are 0.34 and 0.38 , respectively [24]. Noted, the transmitted laser will be absorbed by lower layers of the MTJ. Hence, the energy loss is just related to the reflected laser. This is considered while calculating the energy consumption in the next sections. This energy loss can be reduced by engineering the material and shape of the electrodes. The on-chip laser spot size in nanometer regime can be achieved through vertical cavity surface emitting laser (VCSEL) [25-27]. VCSEL is a type of semiconductor laser diodes. Its unique specification is that, in contrast to the conventional edge-emitting semiconductor lasers, its laser beam is emitted perpendicular to its surface, which makes it a proper candidate for on-chip laser applications including laser-assisted NCS applications. The output power of VCSEL can be tuned through changing the supply voltage of its driver [27]. Hence, a CMOS interfacing circuit is designed in the next section in order to control the laser output power by manipulating the supply voltage.

\section{B. CMOS interfacing circuit}

Fig. 3 (a) shows the block diagram of the proposed LAS-NCS. The spintronic layer includes memristors array, MTJs, control transistors and sensing circuit. The CMOS interface adjusts the output laser power by manipulating the supply voltage of the laser diode driver (LDD). In this way, the CMOS interfacing block can control the MTJ temperature in spintronic layer. Fig. 3 (b) and (c) show the circuit design of the CMOS interfacing block and its timing diagram, respectively. The LAS-NCS operating time can be divided to calibration and processing phases. In the calibration phase, the MTJ temperature is increased from $27^{\circ} \mathrm{C}$ to $127^{\circ} \mathrm{C}$ (first laser pulse with high power) and stabilized at this temperature (second laser pulse with low power). In the processing phase, the MTJ temperature will be kept at $127^{\circ} \mathrm{C}$ with a sequence of low power laser illuminations as shown in Fig. 3 (c). The operation of the CMOS interfacing circuit is as follows. The counter is clocked with a $500 \mathrm{MHz}$ clock and generates the b0, b1 and b2 signals. Then, the logic circuit generates the VLDD signal from the counter's outputs. During the first pulse of $V_{\text {LDD }}$, the level shifter is enabled by a logic circuit and the voltage of VLDD will be set at VDDH that leads to a high output power laser pulse. During the next pulses, the transmission gate is enabled and the level shifter is disabled by the logic circuit. Hence, the voltage of the $\mathrm{V}_{\mathrm{LDD}}$ node is at $\mathrm{V}_{\mathrm{DD}}$ and the laser output power will be low.

\section{SimUlation RESUltS}

The simulations of the LAS-NCS are performed by HSPICE simulator in $65 \mathrm{~nm}$ CMOS technology. The thermal interaction between laser and the MTJ stack is simulated in COMSOL simulator. The laser modeled by the incident heat flux as spatially distributed heat source on the surface. Then, the transient thermal response of the MTJ stack is obtained. The MTJ stack is meshed using a triangular swept mesh. For memristor and MTJ, the threshold adaptive memristor (TEAM) model [28] and the modified MTJ model of [13] (the effect of temperature on MTJ switching is added) are used, respectively.

\section{A. Laser-MTJ interaction}

The laser-MTJ heat transfer is simulated in COMSOL multiphysics for the MTJ stack of [28]. Fig. 4 (a) shows the temperature distribution in MTJ stack at the end of calibration phase, which shows a uniform temperature distribution in the MTJ. Fig. 4 (b) shows the laser power distribution with the power and the diameter of $49 \mu \mathrm{w}$ and $160 \mathrm{~nm}$, respectively. Fig. 4 (c) shows the normalized laser power in each laser radiation during calibration and processing phases. The first two consecutive laser pulses do the calibration phase. The first laser pulse is illuminated for $4 \mathrm{~ns}$ with $100 \%$ power $(49 \mu \mathrm{w})$ to heat the MTJ stack above $127^{\circ} \mathrm{C}$. Then, the laser beam is cut off for $2 \mathrm{~ns}$. The second laser pulse is applied for $4 \mathrm{~ns}$ with $30 \%$ power $(14.7 \mu \mathrm{w})$ in order to stabilize the MTJ temperature. In the processing phase, the laser is illuminated on the MTJ with $30 \%$ power for 2 ns. Then, during the recovery phase, the laser cuts off for 2 ns. This will continue repeatedly to keep the MTJ temperature around $127^{\circ} \mathrm{C}$. Fig 4 (d) shows the maximum and minimum temperature values in the MTJ stack. The maximum temperature of the $\mathrm{MTJ}$ stack is $136^{\circ} \mathrm{C}$, which is sufficiently lower than the Curie temperature of the FL. Besides, the low temperature variations of the MTJ stack $\left( \pm 9^{\circ} \mathrm{C}\right)$ prevents the reliability issues.

\section{B. Delay}

Considering the stochastic nature of switching in MTJs [29], in order to calculate the delay of the MTJ switching, a Monte Carlo (MC) simulation of 1000 iterations is run at different temperatures. Then, the mean value ( $\mu$ Delay) and the standard deviation ( $\sigma$ Delay) of the delay are

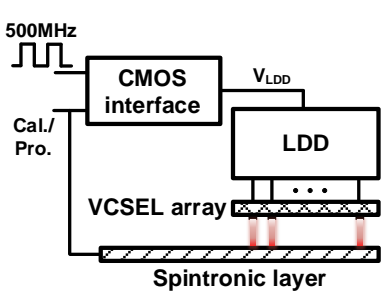

(a)

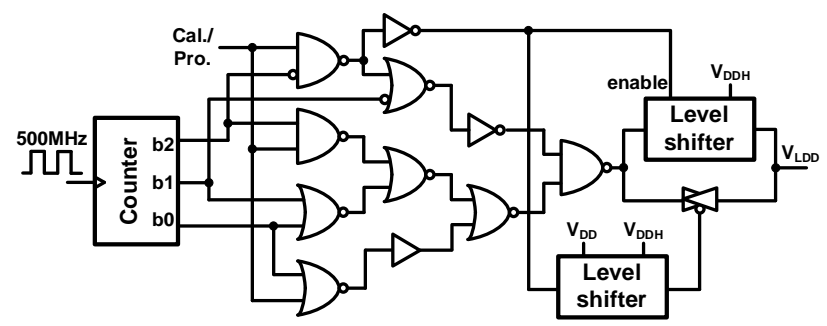

(b)

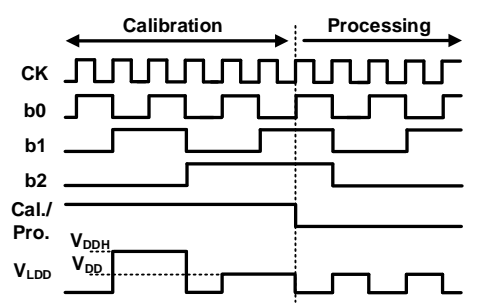

(c)

Fig. 3. (a) The block diagram of the proposed LAS-NCS including CMOS interfacing block, VCSEL array, LDD and the spintronic layer (b) CMOS interfacing circuit design (c) Timing diagram of the CMOS interfacing block. 


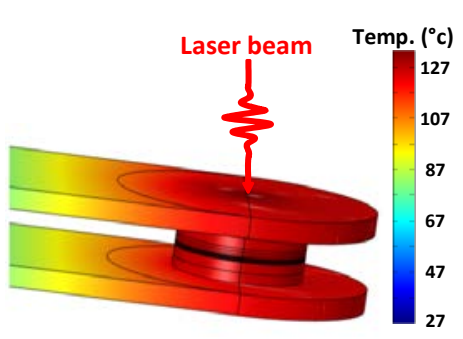

(a)

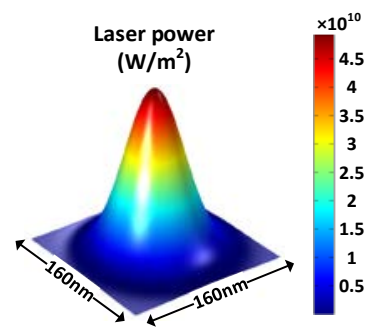

(b)

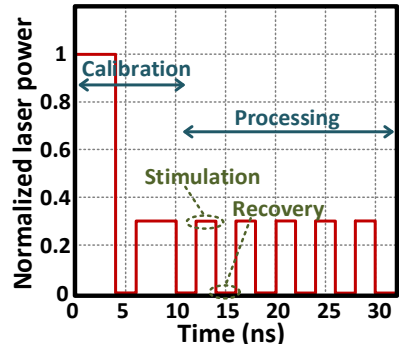

(c)

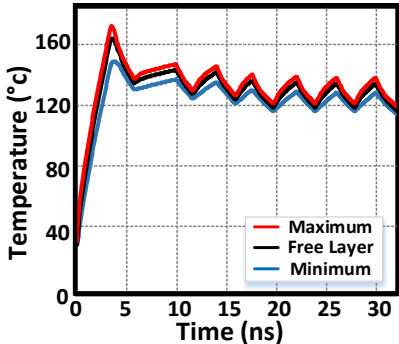

(d)

Fig. 4. (a) The temperature distribution in MTJ stack at the end of calibration phase. (b) The laser power distribution. (c) The laser power pulse normalized to maximum laser power versus time. (d) The maximum and minimum temperature of MTJ stack and the free layer temperature versus time.

calculated. To guarantee the MTJ switching in the worst case, the duration of the stimulation phase should be longer than $\mu \mathrm{AP} \rightarrow \mathrm{P}+6 \times \sigma_{\mathrm{AP} \rightarrow \mathrm{P}}$. Fig. 5 (a) shows the mean value and 6-sigma worst case of the MTJ switching time in stimulation phase versus temperature. The delay is defined as the difference between the time the MTJ voltage is applied and the time at which the FL magnetization switches. Increasing the temperature from $27^{\circ} \mathrm{C}$ to $127^{\circ} \mathrm{C}$ reduces the 6-sigma worst case of MTJ switching delay by $84 \%$ (Fig. 5 (a)). This will lead to a significantly faster operation of the LAS-NCS compared with the typical-NCS. The 6-sigma worst case of MTJ switching time in stimulation and recovery phases at $127^{\circ} \mathrm{C}$ are $1.38 \mathrm{~ns}$ and $1.19 \mathrm{~ns}$, respectively. Hence, a 2ns stimulation and recovery phase duration ensure the MTJ switching in the worst case during both phases.

\section{Energy consumption}

The total energy consumption of the LAS-NCS includes the energies in the spintronic layer (memristors and MTJs), the CMOS interfacing circuit and the laser. Due to the fact that the calibration phase is done just one time at the beginning of the NCS operation, its energy consumption has no significant effect on the total energy consumption of the LAS-NCS and can be ignored. Fig. 5 (b) shows the energy consumption of an MTJ and the memristors connected the MTJ to the inputs in spintronic layer at different temperatures. The energy consumption of MTJ is calculated by summing up the energy consumption of the stimulation (AP- to P-state switching) and the recovery phases ( $\mathrm{P}$ - to $\mathrm{AP}$-state switching). The total energy consumption of MTJ switching decreases by $80 \%$ while increasing the temperature to $127^{\circ} \mathrm{C}$. This is due to the fact that heating up the MTJ reduces its magnetization saturation and effective anisotropy field that tends to keep magnetization direction of FL aligned with the easy axis. Accordingly, the energy consumption of sensing circuit and firing the post NCS are calculated to be around 107fJ.

In order to calculate the energy consumption of memristor array at elevated temperatures, first, the resistance reduction of memristors should be measured at different temperatures. However, the amount of resistance reduction not only depends on temperature, but also is a function of the initial resistance of memristor (weights) and the applied voltage (inputs). Hence, in order to estimate the average resistance reduction of memristor array, first, a 196x10 NCS is designed to recognize the handwritten digits in MATLAB. Then, using MNIST handwritten digits database [30] (size of the pictures is reduced to $14 \times 14$ ), the NCS is trained using 2000 training images and the weights are extracted. Considering the facts that the negative weights cannot be implemented by memristors, the negative weights are considered as zero. In the next step, the weights are mapped to the resistance of memristors in the array. In order to model the effect of temperature increase on the memristor's resistance, an equation is fitted to the experimental results of [22] for each initial conductance from $0.2 \mu \mathrm{S}$ to $21 \mu \mathrm{S}$. Then, the fitted equations are used in MATLAB to determine the resistance of memristors at $127^{\circ} \mathrm{C}$. Finally, 10000 test images have been applied to the modeled NCS in MATLAB and the energy consumption reduction of memristor array is calculated for each test image. The average energy consumption reduction of a column of memristors connecting their corresponding MTJ to inputs is $90 \%$ by increasing the temperature to $127^{\circ} \mathrm{C}$ (Fig. 5 (b)). This is due to the resistance reduction of memristors and faster MTJ switching at elevated temperatures. This means that the current passes through smaller resistances for a shorter time in each stimulation phase.

The energy consumption per stimulation phase of the CMOS interfacing circuit is shown in Fig. 5 (c) at different temperatures. Noted, the energy consumption of the CMOS interfacing circuit can be decreased using low voltage circuit techniques. However, considering the reduction in the energy consumption of spintronic layer $(\sim 5.8 \mathrm{pJ})$, the energy consumption of CMOS interfacing circuit (30fJ at $127^{\circ} \mathrm{C}$ ) has no significant effect on the total energy consumption of the LASNCS. The energy consumption of the laser source is shown in Fig. 5 (c). Increasing the MTJ temperature requires a laser with higher output power. Hence, the energy consumption of the laser increases by increasing the temperature. In stimulation phase, the laser is turned off and it has no energy consumption. In recovery phase, the laser is illuminated for $2 \mathrm{~ns}$ with $30 \%$ power $(14.7 \mu \mathrm{w})$ to keep the temperature of spintronic layer around $127^{\circ} \mathrm{C}$. Hence, the energy consumption of laser per process (stimulation and recovery phase) is estimated as 29.4fJ using COMSOL simulation. Considering the fact that the energy consumption improvement in the spintronic layer is much higher than the energy consumption of the laser and the CMOS

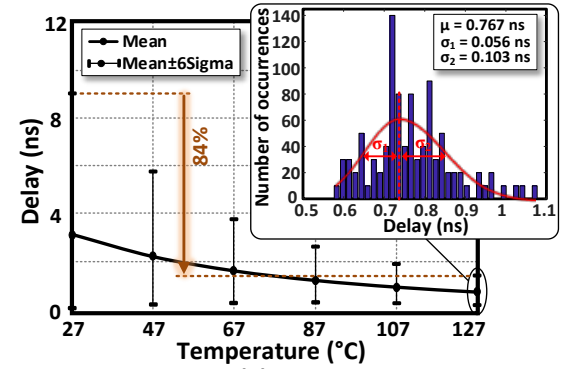

(a)

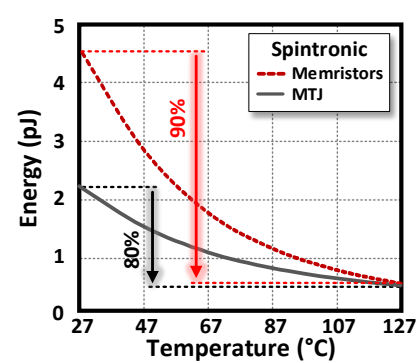

(b)

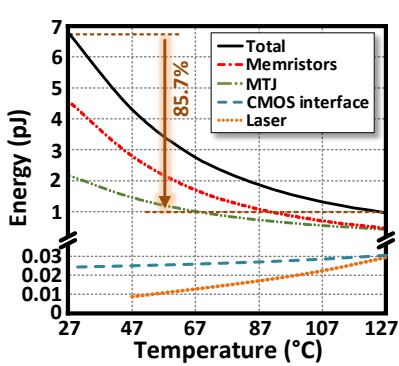

(c)

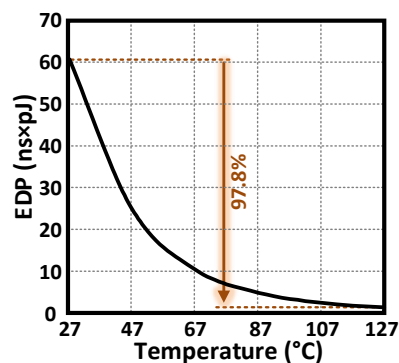

(d)

Fig. 5. (a) The mean value and 6-sigma worst case of MTJ switching delay in stimulation phase, (b) the energy consumption of an MTJ and a column of memristors connecting the MTJ to inputs (c) the total energy consumption of the LAS-NCS (d) the EDP of the LAS-NCS at different temperature from $27^{\circ} \mathrm{C}$ to $127^{\circ} \mathrm{C}$. 
interfacing circuit, the total energy consumption of the LAS-NCS decreases by $85.7 \%$ at $127^{\circ} \mathrm{C}$ compared with $27^{\circ} \mathrm{C}$.

\section{Energy-delay product}

In this section, the energy-delay product (EDP) of the whole LASNCS (photonic layer, CMOS interfacing block and the spintronic layer) are calculated. Considering the fact that the laser illumination is done simultaneously with the stimulation phase, the photonic layer does not affect the delay. Hence, the total delay of the LAS-NCS is remained unchanged. Fig 5 (d) shows the EDP of the LAS-NCS at different temperatures. The EDP of the LAS-NCS is $1.34 \mathrm{~ns} \times \mathrm{pJ}$ at $127^{\circ} \mathrm{C}$, which is improved by $97.8 \%$ compared with the typical NCS at $27^{\circ} \mathrm{C}(60.7 \mathrm{~ns} \times \mathrm{pJ})$. Noted, the Joule heating effect in MTJ due to weighted current from crossbar array is not considered in simulations. The temperature increase due to Joule heating is estimated to be $28^{\circ} \mathrm{C}$ [31], which decreases the laser power and so the total energy consumption of the LAS-NCS.

\section{E. Comparison with the TAS method}

In TAS, in order to heat the FL layer by $200^{\circ} \mathrm{C}\left(>\mathrm{T}_{\mathrm{B}-\mathrm{FL}}\right.$ as mentioned in section II.A) in $5 \mathrm{~ns}$, a current density of $2-4 \times 106 \mathrm{~A} . \mathrm{cm}^{-2}$ with a bias voltage of $1.1 \mathrm{~V}$ is needed for different materials [10]. Hence, the energy consumption of the TAS is estimated as 311fJ-622fJ for the MTJ stack with a cross section area similar to the MTJ stack used in our simulations $\left(\pi \times(30 \mathrm{~nm})^{2}=2.827 \times 10^{-15} \mathrm{~m}^{2}\right)$. The energy consumption of LAS for $200^{\circ} \mathrm{C}$ temperature increase of the MTJ is calculated as 260fJ, which shows 1.2X-2.4X lower energy consumption compared with the TAS. Moreover, the TAS, in its original form, cannot be used with typical MTJs (an antiferromagnet with low $\mathrm{T}_{\mathrm{B}}$ close to the FL is needed). Finally, in TAS, the minimum heating time is limited to 500ps [11]. However, the heating of the ferromagnetic material above Curie temperature in femto-second regime has been shown experimentally by laser [9]. In contrast, the LDD circuit in LAS method can increase its complexity compared with TAS method.

\section{CONCLUSION}

To reduce the energy consumption and the delay of future MTJbased NCSs, a microwatt-nanosecond laser pulse is utilized for the first time to ease the magnetic switching of the MTJ through heating. The energy consumption, delay and energy-delay product of the proposed LAS-NCS are improved by $85.7 \%, 84 \%$ and $97.8 \%$, respectively, at $\mathrm{T}=127^{\circ} \mathrm{C}$ compared with operation at the room temperature. Compared with Joule heating, the proposed laser assisting method consumes 1.2X-2.4X lower energy and can be applied to typical MTJs.

\section{REFERENCES}

[1] U.S. Department of energy, "Neuromorphic computing: from materials to systems architecture," Oct. 2015.

[2] IBM Research: Brain-inspired chip. [online] Available at: http://www.research.ibm.com/articles/brain-chip.shtml

[3] SpiNNaker. [online] Available at: http://apt.cs.manchester.ac.uk/ projects/SpiNNaker/

[4] Europe's Human Brain Project (HBP). [online] Available at: https://www.humanbrainproject.eu/

[5] DeepMind. [online] Available at: https://deepmind.com/

[6] X. Fong et. al. "Spin-transfer torque devices for logic and memory: prospects and perspectives,” IEEE Trans. on Computer-Aided Design of Integrated Circuits and Systems, vol. 35, no. 1, pp. 1-22, Jan. 2016.

[7] S. A. Bender and Y. Tserkovnyak, "Thermally driven spin torques in layered magnetic insulators,” Phys. Rev. B, vol. 93, pp. 064418, Feb. 2016.

[8] C. Safranski et al., "Spin caloritronic nano-oscillator," Nature communications, vol. 8, no. 117, Jul. 2017.
[9] J. Walowski, Physics of laser heated ferromagnets: Ultrafast demagnetization and magneto-Seebeck effect, PhD. Thesis, Niederschsische Staats-und Universittsbibliothek, 2012.

[10] I. L. Prejbeanu et al., "Thermally assisted MRAMs: ultimate scalability and logic functionalities,” Jour. of Phys. D, vol. 46, no. 7, pp. 074002, Jan. 2013.

[11] S. Bandiera and B. Dieny, Thermally assisted MRAMs, In Handbook of Spintronics, Springer: Dordrecht, 2016.

[12] B. Dai et al., "Spin transfer torque switching in exchange coupled amorphous GdFeCo/TbFe bilayers for thermally assisted MRAM application,” Jour. of Phys. D, vol. 50, no. 13, Mar. 2017.

[13] X. Fong et al., Purdue Nanoelectronics Research Laboratory MTJ Model. nanoHUB. (2014), doi:10.4231/D33R0PV04.

[14] N. W. Ashcroft and N. D. Mermin, Solid states physics, Saunders college, 1976.

[15] J. G. Alzate et al., "Temperature dependence of the voltage-controlled perpendicular anistropy in nanoscale $\mathrm{MgO}|\mathrm{CoFeB}| \mathrm{Ta}$ magnetic tunnel junctions,” App. Phys. Let., vol. 104, no. 11, pp. 112410, Mar. 2014.

[16] J. M. Teixeira et al., "Evidence of spin-polarized direct elastic tunneling and onset of superparamagnetism in MgO magnetic tunnel junctions," Phys. Rev. B, vol. 81, no. 13, pp. 134423, Apr. 2010.

[17] Y. Takeuchi et al., "Temperature dependence of energy barrier in CoFeBMgO magnetic tunnel junctions with perpendicular easy axis,” App. Phys. Let., vol. 107, no. 15, pp. 152405, Oct. 2015.

[18] B. Hu et al., "Temperature dependence of spin-dependent tunneling conductance of magnetic tunnel junctions with half-metallic Co2MnSi electrodes,” Phys. Rev. B, vol. 94, pp. 094428, Sep. 2016.

[19] W. F. Brown, "Thermal fluctuations of a single-domain particle," Phys. Rev., vol. 130, no. 5, pp. 1677, Jun. 1963.

[20] J. C. Sankey et al., "Mechanisms limiting the coherence time of spontaneous magnetic oscillations driven by dc spin-polarized currents," Phys. Rev. B, vol. 72, no. 22, pp. 224427, Dec. 2005.

[21] K. Yogendra et al., "Coupled spin-torque nano-oscillator-based computation: a simulation study," ACM journal on emerging technologies in computing systems, vol. 13, no. 4, pp. 56, Jul. 2017.

[22] C. E. Graves et al., "Temperature and field-dependent transport measurements in continuously tunable tantalum oxide memristors expose the dominant state variable,” App. Phys. Let., vol. 110, no. 12, pp. 123501, Mar. 2017.

[23] H. Farkhani et al., "A low-power high-speed spintronics-based neuromorphic computing system using real time tracking method," IEEE Jour. on emerging and selected topics in circuit and systems, vol. 8, no. 3, pp. 627, Mar. 2018.

[24] W. S. M. Werner, K. Glantschnig and C. Ambrosch-Draxl, "Optical constants and inelastic electron-scattering data for 17 elemental metals,” Jour. of Phys. and Chem. Ref. Data, vol. 38, no. 4, pp. 1013, Jan. 2009.

[25] Z. Zhou, B. Yin and J. Michel, "On-chip light sources for silicon photonics," Nature Light: Science \& Applications, vol. 4, e358, Aug. 2015.

[26] C. Chen et al., "Sharing and Placement of On-chip Laser Sources in Silicon-Photonic NoCs,” IEEE/ACM International Symposium on NoCS, pp. 88-95, 2014.

[27] V. Kozlov and A. C. Carusone, "Capacitively-Coupled CMOS VCSEL Driver Circuits," IEEE Jour. of Solid-State Circuits, vol. 51, no. 9, pp. 2077, Sep. 2016.

[28] S. Kvatinsky et al., “TEAM: threshold adaptive memristor model,” IEEE Trans. on Circuits and Systems-I: Regular Papers, vol. 60, no. 1, pp. 211, Jan. 2013.

[29] T. Devolder et al., "Single-shot time-resolved measurements of nanosecond-scale spin-transfer induced switching: stochastic versus deterministic aspects,” Phys. Rev. Let., vol. 100, no. 5, pp. 057206, Feb. 2008.

[30] Y. LeCun, L. Bottou, Y. Bengio, and P. Haffner, "Gradient-based learning applied to document recognition,” Proc. IEEE, vol. 86, no. 11, pp. 22782324, Nov. 1998.

[31] A. Chavent et al., "Steady state and dynamics of Joule heating in magnetic tunnel junctions observed via the temperature dependence of RKKY coupling,” Phys. Rev. App., vol. 6, no. 3, pp. 034003, Sep. 2016. 